\title{
Digital colour matching in restorative dentistry
}

\author{
The use of digital imaging for colour matching and communication in restorative dentistry
}

\author{
F. D. Jarad, M. D. Russell and B. W. Moss Br Dent J 2005; 199: 43-49.
}

Objective

To develop a shade matching method based on digital imaging and to compare observers' ability using this method with the conventional one set against a spectrophotometric 'gold standard'

\section{Methods}

Two Vita Lumin shade guides were used in this study, nine shades being selected from the first Vita Lumin shade guide, A2, A3, A3.5, B2, B3, B4, $\mathrm{C} 1, \mathrm{C} 2$ and $\mathrm{C} 3$. A second shade guide was used to match the selected shades. A Nikon Coolpix 990 digital camera with Nikon SB21B ring flash was used to record the digital images of the shade tabs of the two shade guides and the images were processed using Adobe Photoshop software. A total of 27 samples (three replicates of each shade of the nine tabs selected) were matched with a digital shade guide prepared from the digital images of the second shade guide by 10 observers on a computer screen (computerised matching method). The 10 observers also matched the same shade tabs using the conventional matching method simulated in a phantom head. In addition colour values were obtained from the digital images using Adobe Photoshop and quantified according to an internationally recognised scale as CIELAB colour values, $L^{*}, a^{*}, b^{*}$. The relationship of the digitally derived $C I E L A B$ values to the $C I E L A B$ values obtained using a reflectance spectrophotometer was investigated.

\section{Results}

The cross tabulation statistical analysis showed a statistically significant difference $(p<0.001)$ between the conventional method and the computer method with a $43 \%$ and $61.1 \%$ correct match respectively. There was also a statistically significant difference between observers in both methods ( $p<0.001$ and $p<0.04$ for the conventional and computer methods respectively). A statistically significant high correlation of the CIELAB colour values were found between both colour measurement methods $(p<0.001)$.

\section{Conclusion}

The observers' shade matching performance was significantly better with the computer method compared with the conventional one. There was a large variation in the observers' matching ability. The digital camera can be used as a means of colour measurements in the dental clinic.

\section{IN BRIEF}

- Demonstrates the difficulties of matching teeth and the need for an objective and reliable means of shade matching.

- Digital images acquired under standard lighting conditions can provide reliable colour measurements.

- The use of digital images in shade matching will allow technicians to view variations in tooth colour and improve communication between technicians and clinicians.

\section{COMMENT}

Colour matching and communication with the dental lab is one of the most frustrating challenges for restorative dentists. There is no agreed method of training, a range of shade matching technologies and the quality of feedback to the operator regarding their ability is generally poor. Furthermore, the biological function of colour vision varies between operators, although some dental schools have reported that they screen their students for difficulties in this area.

This paper uses a simple digital photographic set up to address some of these issues. The paper validates the use of digital photographs as a method of communication with the dental lab. Provided there is an image of the shade guide available on the image of the teeth, it appears that a satisfactory evaluation of shades can be obtained from the photograph. In the case of complex tooth colours this is a very valuable method of transferring information to the lab.

The choice of the Vita Lumin shade guide is pragmatic in that it is the most commonly used. It is well-recognised that it has major limitations. The colour range with the newer Vita Master guide is much better distributed across normal tooth colours with a much clearer differentiation between the shades. It would be interesting to see how the use of this improved shade guide would affect the experiment.

Digital cameras incorporate software that manipulates the colour balance and distribution within an image, providing the richly saturated colour images consumers demand. The camera chosen here has such algorithmic processing - in fact Nikon are very much respected for the quality of colour manipulation they use. Professional digital cameras offer an option of recording colour values as registered by the imaging chip - the so-called RAW data. This unmanipulated data would offer a significant advantage over the altered colours with the type of analysis that the authors undertook. A very close correlation between $L A B$ values obtained from the imaging chip and those obtained from the spectrophotometer would be expected.

One customised method of electronic shade recording is examined and the paper is therefore justified in making no reference to the increasing number of commercial digital shading systems on the market. The reader must put this paper into such a context and look at this experiment in relation to such systems, several of which combine spectrophotometric measurement with digital imaging of teeth. There is a high level of variation between the subjects who made the colour evaluations, which can also be a problem. At one end of the scale, one observer managed much better without the computer system. Given this range across operators, it would be informative if the experiment was repeated across a much greater range of operators with a clearer record of their past experience and skill levels.

Electronic colour standards that are commonly found in colour measurement in dentistry are explained and the paper shows how these may be applied experimentally. The paper is a useful introduction for readers and will provide background knowledge for GDPs selecting an electronic shade measurement system for their practice.

Dr N. M. Jedynakiewicz

Lecturer in Restorative Dentistry, The University of Liverpool doi: 10.1038/sj.bdj.4812556 